\title{
Out-of-plane equilibrium points in the restricted three-body problem with oblateness (Research Note)
}

\author{
C. N. Douskos and V. V. Markellos
}

Department of Engineering Sciences, University of Patras, 26500 Patras, Greece

e-mail: [c.douskos; markellos]@des.upatras.gr

Received 14 July 2005 / Accepted 26 September 2005

\section{ABSTRACT}

The equations of motion of the three-dimensional restricted three-body problem with oblateness are found to allow the existence of out-of-plane equilibrium points. These points lie in the $(x-z)$ plane almost directly above and below the center of each oblate primary. Their positions can be determined numerically and are approximated by series expansions. The effects of their existence on the topology of the zero-velocity curves are considered and their stability is explored numerically.

Key words. celestial mechanics

\section{Introduction-equations}

The restricted three-body problem with oblateness of primaries, with or without radiation, has received attention especially in the two-dimensional case and with respect to its five coplanar equilibrium points, i.e. the collinear (or "Eulerian") points $L_{1}, L_{2}, L_{3}$ and the isosceles triangle (or "Lagrangian") points $L_{4}, L_{5}$ (e.g. Sharma \& Subba Rao 1976; Sharma 1987). The three-dimensional case has also been considered (e.g. Sharma \& Subba Rao 1976; Oberti \& Vienne 2003; Gurfil \& Meltzer 2005). However, to the present authors' knowledge no mention has been made of any equilibrium points out of the plane of motion of the primaries for this problem.

In the present paper, using the equations of the threedimensional problem given in the literature we report the existence of equilibrium points located in the $(x-z)$ plane, above and below the center of each oblate primary. We obtain their positions both numerically and by analytical approximations in the form of power series in the oblateness coefficient. The case of oblateness of one or both primaries and the case of oblateness of one primary and radiation of the other are considered. We also consider the effects of their existence on the topology of the zero velocity curves and obtain numerical evidence indicating that these equilibrium points are unstable.

The three-dimensional restricted three-body problem with an oblate primary $m_{2}$ is described in a barycentric rotating coordinate system by the equations of motion:

$\ddot{x}-2 n \dot{y}=\frac{\partial w}{\partial x}, \quad \ddot{y}+2 n \dot{x}=\frac{\partial w}{\partial y}, \quad \ddot{z}=\frac{\partial w}{\partial z}$,
$w=w_{2}=\frac{n^{2}}{2}\left(x^{2}+y^{2}\right)+\frac{1-\mu}{r_{1}}+\frac{\mu}{r_{2}}\left[1+\frac{A_{2}}{2 r_{2}^{2}}\left(1-\frac{3 z^{2}}{r_{2}^{2}}\right)\right]$

(Sharma \& Subba Rao 1976; Oberti \& Vienne 2003) where:

$$
\begin{aligned}
& r_{1}=\sqrt{(x-\mu)^{2}+y^{2}+z^{2}}, \\
& r_{2}=\sqrt{(x-\mu+1)^{2}+y^{2}+z^{2}},
\end{aligned}
$$

are the distances of the massless third body from the primaries, $n=\sqrt{1+3 A_{2} / 2}$ is the angular velocity of the rotating coordinate system, and $A_{2}$ is the oblateness coefficient of $m_{2}$. For values of the mass parameter $\mu=m_{2} /\left(m_{1}+m_{2}\right)<1 / 2$ the oblate primary $m_{2}$ located on the negative $x$-axis at $(-1+\mu, 0)$ is less massive than $m_{1}$, while for $\mu>1 / 2$ the primary $m_{1}$ located at $(\mu, 0)$ is the less massive one. When $m_{1}$ is also oblate, with oblateness coefficient $A_{1}$, the same equations may be used with $w=w_{12}$ :

$$
\begin{aligned}
w_{12}= & \frac{n^{2}}{2}\left(x^{2}+y^{2}\right)+\frac{1-\mu}{r_{1}}\left[1+\frac{A_{1}}{2 r_{1}^{2}}\left(1-\frac{3 z^{2}}{r_{1}^{2}}\right)\right] \\
& +\frac{\mu}{r_{2}}\left[1+\frac{A_{2}}{2 r_{2}^{2}}\left(1-\frac{3 z^{2}}{r_{2}^{2}}\right)\right],
\end{aligned}
$$

where now $n=\sqrt{1+3\left(A_{1}+A_{2}\right) / 2}$. Finally, when only $m_{2}$ is oblate but $m_{1}$ is radiating with radiation coefficient $q_{1}$, Eq. (1) may be used with $w=w_{3}$ :

$w_{3}=\frac{n^{2}}{2}\left(x^{2}+y^{2}\right)+\frac{q_{1}(1-\mu)}{r_{1}}+\frac{\mu}{r_{2}}\left[1+\frac{A_{2}}{2 r_{2}^{2}}\left(1-\frac{3 z^{2}}{r_{2}^{2}}\right)\right]$. 


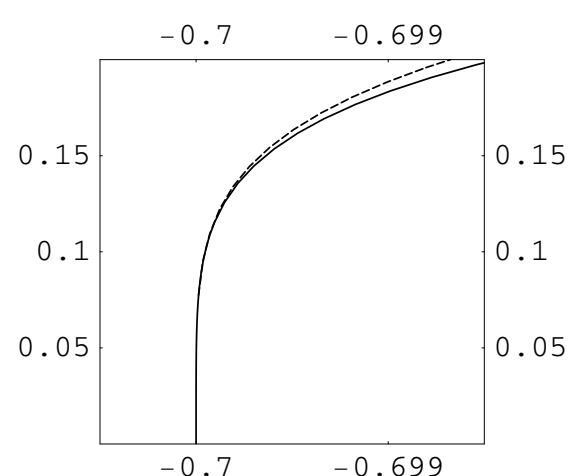

Fig. 1. Position of $L_{z 1}^{(2)}$ in the $(x-z)$ plane as a function of $A_{2}$ in the interval [0,0.02], for $\mu=0.3$.

Equations (1) admit the Jacobi integral:

$2 w-\left(\dot{x}^{2}+\dot{y}^{2}+\dot{z}^{2}\right)=C$,

where $C$ is the Jacobi constant.

\section{Positions of the out-of-plane equilibrium points}

The positions of the out-of-plane equilibrium points can be found from the equations of motion by putting all velocity and acceleration components equal to zero and solving the resulting system, $\frac{\partial w}{\partial x}=\frac{\partial w}{\partial y}=\frac{\partial w}{\partial z}=0$, numerically for $x, y, z$. The second equation is satisfied for $y=0$, so we must solve the remaining two equations for $y=0$ and $z \neq 0$. In the case of $w_{2}$, for example, we have to solve the system:

$$
\begin{aligned}
n^{2} x- & \frac{(1-\mu)(x-\mu)}{r_{10}^{3}} \\
& -\frac{\mu(x-\mu+1)}{r_{20}^{3}}\left(1+\frac{3 A_{2}}{2 r_{20}^{2}}-\frac{15 A_{2} z^{2}}{2 r_{20}^{4}}\right)=0 \\
& -\frac{1-\mu}{r_{10}^{3}}-\frac{\mu}{r_{20}^{3}}\left(1+\frac{9 A_{2}}{2 r_{20}^{2}}-\frac{15 A_{2} z^{2}}{2 r_{20}^{4}}\right)=0,
\end{aligned}
$$

with $r_{10}=\sqrt{(x-\mu)^{2}+z^{2}}, r_{20}=\sqrt{(x-\mu+1)^{2}+z^{2}}$. The outof-plane equilibrium points are found to be located in the $(x-z)$ plane, above and below the center of each oblate primary in symmetrical positions with respect to the $(x-y)$ plane. In the case of both primaries being oblate the positions of the out-ofplane equilibrium points near $m_{2}$, called here $L_{z 1}^{(2)}$ and $L_{z 2}^{(2)}$, have coordinates $\left(x_{0}, 0, \pm z_{0}\right)$ which can be approximated in the form of power series to third-order terms in $A_{2}$ as follows:

$$
\begin{aligned}
x_{0}= & -1+\mu+\frac{9 \sqrt{3}\left(8+45 A_{1}\right)(1-\mu)}{4 \mu} A_{2}^{5 / 2} \\
& +O\left(A_{2}^{7 / 2}\right), \\
z_{0}= & \sqrt{3} \sqrt{A_{2}}-\frac{9\left(2+9 A_{1}\right)(1-\mu)}{4 \mu} A_{2}^{2} \\
& +\frac{81\left(2+25 A_{1}\right)(1-\mu)}{8 \mu} A_{2}^{3}+O\left(A_{2}^{7 / 2}\right) .
\end{aligned}
$$

By means of the transformation: $\mu \rightarrow 1-\mu, A_{2} \rightarrow A_{1}, A_{1} \rightarrow$ $A_{2}$, these expansions give the positions of the two equilibrium points above and below $m_{1}$, which we call $L_{z 1}^{(1)}, L_{z 2}^{(1)}$.
Similarly, in the case of an oblate primary $m_{2}$ and a radiating primary $m_{1}$ the positions of $L_{z 1}^{(2)}$ and $L_{z 2}^{(2)}$ are approximated to third-order terms in $A_{2}$ by:

$$
\begin{aligned}
x_{0}= & -1+\mu+\frac{3 \sqrt{3}\left(1-q_{1}\right)(1-\mu)}{\mu} A_{2}^{3 / 2} \\
& +\frac{9 \sqrt{3}\left(1+3 q_{1}\right)(1-\mu)}{2 \mu} A_{2}^{5 / 2} \\
& -\frac{27\left(1-q_{1}\right)(1-\mu)\left[2+11(1-\mu) q_{1}\right]}{2 \mu^{2}} A_{2}^{3} \\
& +O\left(A_{2}^{7 / 2}\right), \\
z_{0}= & \sqrt{3} \sqrt{A_{2}}-\frac{9 q_{1}(1-\mu)}{2 \mu} A_{2}^{2} \\
& -\frac{63 \sqrt{3}\left(1-q_{1}\right)^{2}(1-\mu)^{2}}{4 \mu^{2}} A_{2}^{5 / 2} \\
& +\frac{81 q_{1}(1-\mu)}{4 \mu} A_{2}^{3}+O\left(A_{2}^{7 / 2}\right) .
\end{aligned}
$$

In both the above approximations higher order terms can be obtained by solving the relevant equations, e.g. by Newton's method using the software package Mathematica. We note that the use of $x_{0}=-1+\mu, z_{0}=\sqrt{3} \sqrt{A_{2}}$, as initial approximations allows the accurate, analytical-approximate or numerical, determination of the position of $L_{z 1}^{(2)}$.

In the following we consider the case when only the primary $m_{2}$ is oblate $\left(A_{2} \neq 0, A_{1}=0\right)$ and the other primary is not radiating $\left(q_{1}=1\right)$. In this case both the above approximations reduce to:

$$
\begin{aligned}
& x_{0}=-1+\mu+\frac{18 \sqrt{3}(1-\mu) A_{2}^{5 / 2}}{\mu}+O\left(A_{2}^{7 / 2}\right) \\
& z_{0}=\sqrt{3} \sqrt{A_{2}}-\frac{9(1-\mu) A_{2}^{2}}{2 \mu}\left(1-\frac{9 A_{2}}{2}\right)+O\left(A_{2}^{7 / 2}\right) .
\end{aligned}
$$

As an example we give in Fig. 1 the position of $L_{z 1}^{(2)}$ in the $(x-z)$ plane as a function of $A_{2}$ in the interval $[0,0.02]$ for $\mu=0.3$, as obtained from (10) (continuous line) and as found numerically (broken line). Note the different scales for the $x$-axis (horizontal) and the $z$-axis (vertical). This illustrates that the equilibrium point lies almost exactly above the center of the oblate primary $m_{2}$ located at $(-0.7,0)$.

\section{Zero-velocity curves in the $(x-z)$ plane}

Oblateness of primaries and the existence of the out-of-plane equilibrium points has significant effects on the structure of the regions allowed to motion and their boundaries, the zerovelocity surfaces. In this section we present the zero-velocity curves in the $(x-z)$ plane in the case of $w_{2}$. As a particular example we show in Fig. 2 the four possible topologies of the curves in this case for $\mu=0.3$. Only the curves for Jacobi constant values corresponding to the collinear equilibrium points and the out-of-plane point $L_{z 1}^{(2)}\left(L_{z 2}^{(2)}\right)$ are shown. For clarity, in each case we also show separately (left frame in each row) the curve corresponding to the out-of-plane equilibrium point. These four topologies correspond to four successive intervals 

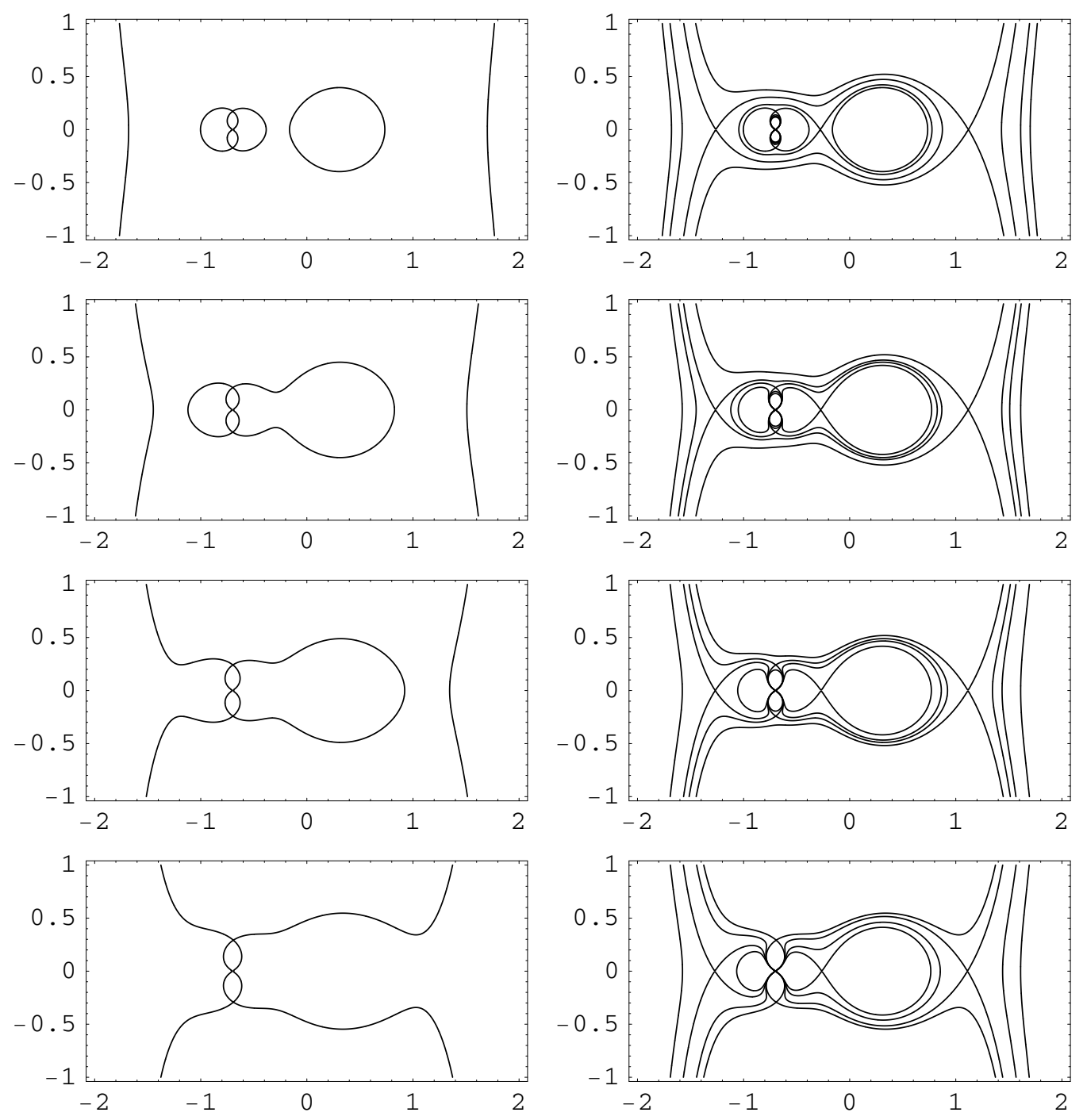

Fig. 2. Zero-velocity curves in the $(x-z)$ plane for $\mu=0.3$ and $A_{2}=0.01,0.015,0.02,0.03$ (top to bottom).

of $A_{2}$ values, separated by three transition values at which a change of topology occurs. These transitions occur when the Jacobi constant value corresponding to the out-of-plane point coincides with one of the values corresponding to the collinear equilibrium points. In all cases, between the center of the oblate primary and its companion out-of-plane equilibrium points the zero-velocity curves form small ovals of regions not allowed to motion.

\section{Stability}

To determine the linear stability of the out-of-plane point $L_{z 1}^{(2)}$ we transfer the origin to $\left(x_{0}, 0, z_{0}\right)$ and linearize the equations of motion, obtaining:

$$
\begin{aligned}
& \ddot{x}-2 n \dot{y}=w_{x x} x+w_{x z} z, \\
& \ddot{y}+2 n \dot{x}=w_{y y} y, \\
& \ddot{z}=w_{z x} x+w_{z z} z,
\end{aligned}
$$

where the partial derivatives are evaluated at the equilibrium point, and $w_{z x}=w_{x z}$. In the present case we also have $w_{x x}+w_{y y}+w_{z z}=2 n^{2}$. Stability of $L_{z 1}^{(2)}$ is determined by the roots of the characteristic polynomial:

$$
\begin{gathered}
\lambda^{6}+2 n^{2} \lambda^{4}+\left(w_{x x} w_{y y}+w_{x x} w_{z z}+w_{y y} w_{z z}-w_{x z}^{2}-4 n^{2} w_{z z}\right) \lambda^{2} \\
+w_{y y}\left(w_{x z}^{2}-w_{x x} w_{z z}\right)
\end{gathered}
$$

We have computed these roots in the case of $w_{2}$ for a wide range of $\mu$ and $A_{2}$ values and found no case in which the roots are all imaginary. This provides numerical evidence that the out-of-plane equilibrium points are generally unstable.

\section{Conclusions}

We have found that the equations of motion of the threedimensional restricted three-body problem with oblateness given in the literature allow the existence of out-of-plane equilibrium points. These points lie in the $(x-z)$ plane almost directly above and below the center of each oblate primary. Their positions are determined numerically and are approximated by series expansions in the oblateness coefficient. Oblateness and the existence of the out-of-plane equilibrium points is seen to have significant effects on the topology of the zero-velocity 
curves in the $(x-z)$ plane. In particular, between the center of the oblate primary and its companion out-of-plane equilibrium points the zero-velocity curves form small ovals of regions not allowed to motion. Finally, numerical evidence is obtained indicating that these equilibrium points are unstable. We have not checked, however, if their existence might be due to the truncation of the potential employed in deriving the equations of motion.

\section{References}

Gurfil, P., \& Meltzer, D. 2005, 25th Israeli Annual Conference on Aerospace Sciences, preprint

Oberti, P., \& Vienne, A. 2003, A\&A, 397, 353

Sharma, R. K. 1987, Ap\&SS, 135, 271

Sharma, R. K., \& Subba Rao, P. V. 1976, Celes. Mech., 13, 137 